\title{
SOME APPLICATIONS OF THE RAYLEIGH-RITZ METHOD TO THE THEORY OF THE STRUCTURE OF MATTER ${ }^{1}$
}

HUBERT M. JAMES

I have been asked to discuss for you some applications of the Rayleigh-Ritz method in physics. Rather than attempt to survey the whole of a discouragingly large field I have preferred to restrict my attention to some few topics from the theory of atomic and molecular structure. The application of the Rayleigh-Ritz method in this field has been carried out with some care and persistence, and there are enough results available to make possible a critical discussion of the potentialities and limitations of the method. This field also presents good examples of how one's mathematical procedure is influenced by one's physical notions concerning the system to be studied, and, in turn, what sort of physical conclusions may be drawn from results yielded by the method.

First of all I shall indicate why recourse to the Ritz ${ }^{2}$ method is necessary if one is to obtain a satisfactorily accurate treatment of some of the principal problems in atomic and molecular theory. I shall indicate, though not in as much detail as I should like, the nature of the considerations by which one chooses the set of coordinate functions employed in this method, and show how rapid a convergence one can hope for in the approach to the solution. After presentation of some of the very satisfactory results one can obtain, by the application of enough hard work, in not too unfavorable cases, I shall finally have to indicate the increasingly serious limitations of the method as one seeks to apply it to the treatment of more and more complicated physical systems.

The fundamental mathematical problem in the study of an atomic system by wave mechanics is usually the solution of the stationary state wave equation for that system. In the non-relativistic case this is a linear partial differential equation of the second order, which can be symbolized thus:

$$
H \psi=E \psi
$$

Here $H$ represents the Hamiltonian operator for the system obtained

1 An address delivered before the meeting of the Society in Washington, D. C., on May 3, 1941, by invitation of the Program Committee.

${ }^{2}$ W. Ritz, Journal für die reine und angewandte Mathematik, vol. 135 (1909), p. 1. 
from the Hamiltonian function $H$ of classical mechanics by the operator substitution

$$
p_{i} \rightarrow \frac{h}{2 \pi i} \frac{\partial}{\partial q_{i}}
$$

where $h$ is Planck's constant. For instance, for a hydrogen atom with fixed nucleus $H$ is the sum of the kinetic energy of the one electron and the potential energy of the electron with respect to the nucleus:

$$
H=\frac{1}{2 m}\left(p_{x}^{2}+p_{y}^{2}+p_{z}^{2}\right)-\frac{e^{2}}{r} .
$$

The Hamiltonian operator correspondingly involves the Laplacian operator in a term representing the kinetic energy of the electron and a scalar multiplier representing the potential energy:

$$
H=-\frac{h^{2}}{8 \pi^{2} m} \nabla^{2}-\frac{e^{2}}{r} .
$$

The wave equation is then

$$
-\frac{h^{2}}{8 \pi^{2} m} \nabla^{2} \psi-\frac{e^{2}}{r} \psi=E \psi .
$$

In these equations $E$ is a number, the energy of the system, and $\psi$, the corresponding solution, is the wave function which describes the state of the system when it has that energy. $\psi$ is obviously a function of all the spatial coordinates needed to define a configuration of the system. In the case of a simple molecule like $\mathrm{Cl}_{2}$, for instance, $\psi$ would depend on 108 variables. It is this multiplicity of variables which makes it so hard for physicists to interest mathematicians in some problems they would like very much to have solved.

Now not all the solutions of the wave equation are of interest, but only those which can be physically interpreted. This requirement of physical interpretability places certain auxiliary conditions on the functions $\psi$, of ten merely that they be quadratically integrable or that they remain bounded. Between the physically interpretable wave functions and the stationary states in which the system may be found there is a one-to-one correspondence. Thus in many cases physically satisfactory solutions of the wave equation exist only for certain discrete values of $E$, and in such cases one finds that the physical system also can possess only these discrete energies.

The physically satisfactory solutions $\psi$ of such an equation and the 
corresponding energies $E$ form the raw material for any detailed quantitative treatment of the atomic system. For instance, a molecule can emit radiation when its energy changes from one of the discrete possible values to another. One can compute the average intensity of such radiation as soon as one knows the $\psi$ functions for the initial and final stationary states, while the wavelength can be determined at once from the corresponding $E$ 's. Thus by determining all the physically acceptable $\psi$ 's and $E$ 's which satisfy the wave equation for a molecule one could make a complete prediction of the wavelengths and intensities in the spectrum emitted by a gas consisting of these molecules, under any specified conditions. These wave functions are similarly fundamental in the study of collisions and other interactions between atoms or molecules, the absorption and scattering of radiation, and so on. The difficulty is to get reasonably accurate $\psi$ functions on which to base further work.

In the simplest case of the hydrogen atom a complete solution of the problem is possible. The wave equation is separable in spherical coordinates, and one has to deal only with ordinary differential equations which offer no special difficulty. However, when one passes to the next more complicated problem, the helium atom with a heavy nucleus and two electrons, one meets most of the difficulties characteristic of this work.

If we treat the nucleus of the atom as fixed, the wave equation includes terms representing the kinetic energies of the electrons, the potential energies of the first and second electrons with respect to the nucleus, and their potential energy with respect to each other:

$$
\begin{aligned}
&-\frac{h^{2}}{8 \pi^{2} m}\left(\nabla_{1}^{2}+\nabla_{2}^{2}\right) \psi\left(\boldsymbol{r}_{1},\right.\left.\boldsymbol{r}_{2}\right)+\left(-\frac{2 e^{2}}{r_{1}}-\frac{2 e^{2}}{r_{2}}+\frac{e^{2}}{r_{12}}\right) \psi\left(\boldsymbol{r}_{1}, \boldsymbol{r}_{2}\right) \\
&=E \psi\left(\boldsymbol{r}_{1}, \boldsymbol{r}_{2}\right) .
\end{aligned}
$$

It involves six independent variables or two vectors $\boldsymbol{r}_{1}, \boldsymbol{r}_{2}$, defining the positions of the two electrons; one might, for instance, take as three of these variables the separations $r_{1}, r_{2}, r_{12}$ of the three particles, on which the potential energy terms depend, and as the other three variables the angles necessary to define the orientation in space of the triangle formed by the three particles.

The fundamental difficulty of this equation arises from the fact that it is not completely separable. One can separate out the angle variables just mentioned, but there remains a partial differential equation in three variables which is not separable in any set of coordinates. In the very simplest case, in which $\psi$ does not depend on the angle variables at all, the separated equation becomes 


$$
\begin{gathered}
-\frac{h^{2}}{8 \pi^{2} m}\left(\frac{\partial^{2}}{\partial r_{1}^{2}}+\frac{\partial^{2}}{\partial r_{2}^{2}}+2 \frac{\partial^{2}}{\partial r_{12}^{2}}+\frac{2}{r_{1}} \frac{\partial}{\partial r_{1}}+\frac{2}{r_{2}} \frac{\partial}{\partial r_{2}}+\frac{4}{r_{12}} \frac{\partial}{\partial r_{12}}\right. \\
\left.+\frac{r_{1}^{2}-r_{2}^{2}+r_{12}^{2}}{r_{1} r_{12}} \frac{\partial^{2}}{\partial r_{1} \partial r_{12}}+\frac{r_{2}^{2}-r_{1}^{2}+r_{12}^{2}}{r_{2} r_{12}} \frac{\partial^{2}}{\partial r_{2} \partial r_{12}}\right) \psi \\
+\left(-\frac{2 e^{2}}{r_{1}}-\frac{2 e^{2}}{r_{2}}+\frac{e^{2}}{r_{1}}\right) \psi=E \psi .
\end{gathered}
$$

This equation is not only complicated, but unpleasantly singular, the potential energy becoming infinite as any one of the three variables approaches zero. Though it does not seem outside the range of possibility, no one has yet been able to set up a direct method for solving this equation or even for proving the existence of solutions. One must immediately resort to approximations.

One method by which physicists often deal with such equations is that of forcing a separation of variables by neglect of judiciously chosen small terms, the resulting ordinary differential equations then being treated by the customary methods. This and the closely related methods of Hartree ${ }^{3}$ and Fock ${ }^{4}$ actually serve to give useful approximate solutions of atomic wave equations involving several hundreds of variables. Peculiarly enough, the limitations of these methods are in practice apt to be more serious the lighter, the less complicated, the atom.

In the case of the state of helium with lowest energy, the ground state, computations by this method become quite simple. If one makes the very crude approximation of neglecting the mutual repulsion of the electrons, as represented by the term $e^{2} / r_{12}$, the approximate wave function becomes

$$
\psi=C e^{-2\left(r_{1}+r_{2}\right)},
$$

the unit of length being the atomic unit $a_{H}=5.28 \times 10^{-9} \mathrm{~cm}$. The atomic energy computed with this function is -76.43 electron volts, against an observed value of -80.62 electron volts. Neglect of a group of terms,

$$
\frac{e^{2}}{r_{12}}-\frac{5}{32}\left(\frac{e^{2}}{r_{1}}+\frac{e^{2}}{r_{2}}\right),
$$

${ }^{3}$ D. R. Hartree, Proceedings of the Cambridge Philosophical Society, vol. 24 (1928), p. 189; J. C. Slater, Physical Review, vol. 32 (1928), p. 339. Results published in many papers of Hartree, mostly in Proceedings of the Royal Society of London, series A.

${ }^{4}$ V. Fock, Zeitschrift für Physik, vol. 61 (1930), p. 126. 
which is on the average smaller than the single term $e^{2} / r_{12}$, leads to a better wave function, $\psi=C e^{-(27 / 16)\left(r_{1}+r_{2}\right)}$, and a computed atomic energy in error by 1.53 electron volts. Further improvements are not easily obtained, and by pressing this method to its limit one can reduce the error in the computed energy only to 0.81 electron volts.

To obtain wave functions and energy values more accurate than these, as one very much wants to do, one must resort to the variational formulation of the problem. One then seeks to determine the wave functions, not as solutions of a differential equation, but as the properly continuous functions which extremalize the average energy

$$
\bar{E}=\frac{\int \psi^{*} H \psi d \tau}{\int \psi^{*} \psi d \tau}
$$

the corresponding extremal values of this quantity are then the stationary state energies of the system, which one wants to determine.

A variety of variational procedures have been applied in the theory of atomic structure, particularly in the study of the lowest energy states of the systems. The correct wave function for such a state makes the computed energy an absolute minimum. An approximate wave function will then presumably be better the lower-that is, the more nearly correct-the corresponding energy. It is accordingly a direct, and very common, procedure to set up approximate wave functions known to have a generally appropriate form, but involving adjustable parameters $\alpha, \beta, \cdots$. Adjusting these parameters to minimize the average energy, one obtains the best approximate wave function of the assumed form, and possesses in the minimum value of $\bar{E}$ the most favorable upper limit on the ground state energy of the system.

For instance, one might assume a function of the form given by a forced separation of variables, but treat the constant in the exponential, which is the effective charge of the nucleus, as a variable parameter:

$$
\psi\left(\alpha ; \boldsymbol{r}_{1}, \boldsymbol{r}_{2}\right)=C e^{-\alpha\left(r_{1}+r_{2}\right)} .
$$

The best value of the parameter $\alpha$ is $27 / 16$, the energy error being then 1.53 electron volts. Or one may set up the more elaborate and more flexible function,

$$
\psi\left(\alpha, \beta ; \boldsymbol{r}_{1}, \boldsymbol{r}_{2}\right)=C\left(e^{-\alpha r_{1}} e^{-\beta r_{2}}+e^{-\beta r_{1}} e^{-\alpha r_{2}}\right) .
$$

The best values of the parameters are then $\alpha=2.15, \beta=1.19$, and the error in the computed energy is only 0.76 electron volts, less than that given by any forced separation of variables. 
Such schemes for the variation of parameters are often useful, but they have one serious limitation. In general an excessive amount of labor is required to determine the best values of the parameters, particularly if these occur in exponentials. As a result, the number of parameters which can be handled, and thus the flexibility of the trial functions, is seriously limited. This is one of the points at which the Rayleigh-Ritz method is superior to all others. Without excessive labor one can deal with Ritz functions containing as many as twenty parameters, whereas with other schemes one is in practice limited to two or three. The result is that the Ritz method is quite without a rival in the accurate computation of wave functions and atomic energies-as distinguished from roughly approximate computations.

The critical step in the application of the Ritz method is the choice of the set of coordinate functions in terms of which the wave functions are to be expressed as sums. Here one must balance against the need for reasonable ease in the computations the requirement of a general suitability in the character of the functions chosen, for even the considerable flexibility of the Ritz function in practice is not sufficient to compensate an inappropriate choice of the form of the component terms. One must bring to bear on this problem all the physical considerations which may be available. In the case of the ground state of helium the relatively simple arguments would run as follows:

First of all, since the electrons are identical the Hamiltonian operator is symmetrical to interchange of their coordinates. From this it follows easily that every $\mathrm{He}$ wave function should be either symmetric or antisymmetric to interchange of the coordinates of the electrons. States of the atom described by symmetric functions are those which the spectroscopist would class as singlet states, while the others are triplet states. The ground state of helium is known to be a singlet state; hence in attempting to determine its wave function one need consider only functions symmetric to interchange of the electrons. Similar general arguments, ${ }^{5}$ combined with the spectroscopic observation that the ground state of helium is an $S$ state, show that this wave function does not depend at all on the angle variables, but depends only on the distances between the particles.

There are other arguments of a less precise character. For instance, if either electron of the atom is very far away from the nucleus the chance is overwhelming that the second electron will be, relatively, very near to it. The electric field at the first electron would then be nearly the same as if the second electron and the nucleus were super-

5 G. Breit, Physical Review, vol. 35 (1930), p. 569. 
imposed-that is, almost Coulomb in character. Now we know that if the field were exactly Coulomb the wave function would decrease exponentially at large distances. Hence we can conclude that the situation will be similar in helium; the wave function should decrease approximately exponentially as the distance of either electron from the nucleus becomes very great.

This fact would be indicated by the approximate wave functions given by any of the methods previously discussed. But there is another important feature of the correct function which none of those approximate functions possesses; not one of them depends on the distance between the electrons. This is physically quite unreasonable, for it implies that the probability of any configuration of the system depends only on the electron-to-nucleus distances, and is just the same whether the electrons are very close together, on the same side of the nucleus, or far apart, on opposite sides of it. Obviously the mutual repulsion of the electrons will tend to keep them away from each other; correspondingly the wave function must tend to decrease in magnitude with decreasing separation of the electrons. It is the inability of most approximate wave functions to take account of this feature in the behavior of the system that is responsible for their largest errors.

How then shall one choose the terms from which the Ritz function is to be constructed? Formally, the simplest choice would be to make the Ritz function a power series, with the typical term,

$$
\underset{r_{1}}{m} r_{2}^{n} r_{12}^{p}
$$

This is impossible, however, for no finite sum of such terms would show the desired exponential decrease for very large $r_{1}$ and $r_{2}$; the very integrals in terms of which the variational procedure is stated would not converge. But one can obtain the desired asymptotic character of the function, and assure the existence of all relevant integrals, by introducing an exponential factor into each term:

$$
e^{-\delta\left(r_{1}+r_{2}\right)}{ }_{r_{1}}^{m} r_{2}^{n} r_{12}^{p}
$$

Introduction of an exponential factor in $r_{12}$ might seem appropriate, but it would be quite unnecessary, and would only make the computations more tedious. A sum of such terms, in addition to the linear parameters characteristic of the Ritz method, would contain an exponential parameter for which it would be much more difficult to determine the very best value. Fortunately in practice one need worry little about the exact value assigned to such parameters; they may 
be fixed once for all by an exploratory computation with a very few terms, or assigned a generally appropriate constant value on the basis of physical considerations of effective nuclear charges.

A finite sum of such terms can have all the properties one desires in respect to flexibility, asymptotic behavior and simplicity, but may lack the proper symmetry to interchange of the coordinates of the electrons. This symmetry one can obtain in various ways. For instance, one may construct the terms from powers of $r_{1}+r_{2}$ and $r_{1}-r_{2}$ :

$$
e^{-\delta\left(r_{1}+r_{2}\right)}\left(r_{1}+r_{2}\right)^{m}\left(r_{1}-r_{2}\right)^{n} r_{12}^{p},
$$

and assure the desired symmetry by including terms with all powers of $\left(r_{1}+r_{2}\right)$, but only even powers of $r_{1}-r_{2}$. These were just the coordinate functions used by Hylleraas in his pioneer work on the theory of the helium atom. ${ }^{6}$

In his first work Hylleraas obtained a quite simple six-term function,

$$
\begin{aligned}
\psi=e^{-1.818\left(r_{1}+r_{2}\right)}\left\{c_{0}+c_{1} r_{12}+c_{2} r_{12}^{2}+c_{3}\left(r_{1}\right.\right. & \left.+r_{2}\right) \\
& \left.+c_{4}\left(r_{1}+r_{2}\right)^{2}+c_{5}\left(r_{1}-r_{2}\right)^{2}\right\} .
\end{aligned}
$$

In the atomic units $R_{H e} h$ the energy computed with this function is -5.80633 , against the observed value of $-5.80736 \pm .00012$; the error of .0139 electron volts is about one-sixtieth that of the best previous computation. In later work ${ }^{7}$ with an eight-term function the computed energy was depressed to -5.80748 . It might seem embarrassing to the theory that the computed upper limit on the energy should come below the observed energy. However, the wave equation treated by Hylleraas involved certain approximations, neglecting relativistic effects and in part, the motion of the nucleus. Adding a correction of +0.00010 for the effect of nuclear motion and one of +0.00018 for relativistic effects ${ }^{8}$ one obtains a theoretical value of -5.80720 for the ground state energy of helium. This agrees with the observed value to one part in 30,000 , practically within the accuracy of observation, and, as was to be expected, lies slightly too high.

This very satisfactory treatment of the three-body problem in wave mechanics is of historical interest in that it gave the first quantitative check on the present theory of atomic structure as applied to systems more complicated than the hydrogen atom. Many theories have been devised to account for the very simple properties of hydro-

${ }^{6}$ E. A. Hylleraas, Zeitschrift für Physik, vol. 54 (1929), p. 347.

${ }^{7}$ E. A. Hylleraas, Zeitschrift für Physik, vol. 65 (1930), p. 209.

${ }^{8} \mathrm{H}$. Bethe, Handbuch der Physik, XXIV/1. See pages 371, 383. 
gen, but no other one of them has been able to stand the test of extension to the treatment of helium. The confidence of the physicist in the essential correctness of the present atomic theory is of course based on a wide variety of comparisons with experiment. A number of other equally impressive examples of this particular quantitative type have been obtained through application of the Ritz method, and will be presented later.

By making use of the observed energies of the higher stationary states of helium one can fix an upper limit on the error of these wave functions. ${ }^{9}$ One can show, for instance, that the root-mean-square error in the six-term function does not exceed 3 per cent; a reasonable estimate is 1 per cent. For the more complicated function the error is of the order of $1 / 5$ per cent. Such accuracy is more than adequate for the purposes of the physicist.

Similar results can be obtained in studies of the lower excited states of helium. These states differ from the ground state in one important physical respect. In the ground state the two electrons are said to be equivalent; the most probable configurations of the atom are those in which the two electrons are at about the same distance from the nucleus. In the lower excited states of the atom just one of the electrons is excited; the most probable configurations are those in which one electron is much farther from the nucleus than the other. The effective nuclear charges are then different for the two electrons, that of the outer electron being reduced by its repulsion by the inner electron. This one should take into account in constructing the Ritz function, replacing

$$
e^{-\delta\left(r_{1}+r_{2}\right) r_{1}^{m} r_{2}^{n} r_{12}^{p}}
$$

by

$$
e^{-\delta_{1} r_{1}} e^{-\delta_{2} r_{2}{ }^{m} r_{1}^{n} r_{1}^{n} r_{12}^{p} .}
$$

The terms in the Ritz function are then to be constructed by making appropriate linear combinations of these functions, symmetric in the case of ${ }^{1} S$ terms, and antisymmetric in the case of ${ }^{3} S$ terms.

If the excited state to be considered is not the lowest of its symmetry one does not obtain the energy of the state as the lowest root of a secular equation, but as the second lowest root, third lowest root, and so on. Fortunately one can show that an approximate energy thus computed is always an upper limit for the energy of the corresponding state of the atom, approaching the correct value from above as the

${ }^{9}$ H. M. James and A. S. Coolidge, Physical Review, vol. 51 (1937), p. 860. 
flexibility and accuracy of the Ritz function are increased. ${ }^{10,11}$ Consequently the error in the computed energy provides an indication (which can be made quantitative) of the accuracy of the corresponding function. This is an outstanding advantage of the Ritz method over all other practical methods of treating excited states, in which an indifferent approximate wave function can by chance lead to the correct value of the energy.

Among the results of computations on excited states of helium are the following: The lowest excited state of helium is a ${ }^{3} S$ state. The observed energy is -4.3505 in the atomic units $R_{H_{\theta}} h$ while Hylleraas and Undheim, ${ }^{10}$ using a six-term function, have computed an energy of -4.3504 , an error of about one part in 40,000. The second excited state is a ${ }^{1} S$ state, with an observed energy of -4.2920 , while Coolidge and James ${ }^{12}$ have used an eleven-term function to compute the value -4.2916 , in error by one part in 10,000 . The greater complexity of the function needed in the case of the more highly excited state will illustrate a general feature of computations with the Ritz method: convergence on the correct function becomes slower the more highly excited is the state considered. This factor, together with the increasing usefulness of certain approximate methods, limits the interest in extending Ritz computations to very highly excited states.

Another simple and important problem is that of the hydrogen molecule, $\mathrm{H}_{2}$. This provides illustrations of the results obtainable with the Ritz method in rather different and more complicated circumstances, and also material for some general remarks about the method, which can here be based on more extensive studies than in the case of helium.

The problem of treating $\mathrm{H}_{2}$, a system containing two heavy nuclei and two light electrons, can be reduced to that of solving the wave equation for the motion of the electrons in the field of the nuclei, considered as fixed. The wave equation is then

$$
\begin{gathered}
\left\{-\frac{h^{2}}{8 \pi^{2} m}\left(\nabla_{1}^{2}+\nabla_{2}^{2}\right)+\left(-\frac{e^{2}}{r_{1}}-\frac{e^{2}}{s_{1}}-\frac{e^{2}}{r_{2}}-\frac{e^{2}}{s_{2}}+\frac{e^{2}}{r_{12}}+\frac{e^{2}}{R}\right)\right\} \psi\left(R ; \boldsymbol{r}_{1}, \boldsymbol{r}_{2}\right) \\
=E(R) \psi\left(R ; \boldsymbol{r}_{1}, \boldsymbol{r}_{2}\right) .
\end{gathered}
$$

This partial differential equation involves the six coordinates of the two electrons as independent variables, and the fixed separation of the nuclei, $R$, as a parameter. It contains terms corresponding to the

\footnotetext{
${ }^{10}$ E. A. Hylleraas and B. Undheim, Zeitschrift für Physik, vol. 65 (1930), p. 759.

11 J. K. L. MacDonald, Physical Review, vol. 43 (1933), p. 830.

12 A. S. Coolidge and H. M. James, Physical Review, vol. 49 (1936), p. 676.
} 
kinetic energies of the first and second electrons, the potential energy of the first electron at a distance $r_{1}$ from the first nucleus and $s_{1}$ from the second nucleus, the potential energy of the second electron in the field of the nuclei, the potential energy of repulsion of the two electrons, and the potential energy of repulsion of the two nuclei. Again the difficulty in solving the wave equation arises from the non-separability of the variables. In this case at most one variable can be separated out, and every exact solution depends on at least five variables -for instance, $r_{1}, r_{2}, s_{1}, s_{2}, r_{12}$.

The physical considerations on which one can base approximate solutions of this equation are interesting but so extensive and complicated that I cannot undertake to present them here. I can suggest only one of several possible lines of thought. If, for instance, one neglects the repulsion of the electrons the problem becomes completely separable. To this approximation, each electron moves as it would in the absence of the second electron-that is, as if it were the one electron of an ionized hydrogen molecule. Correspondingly the wave function becomes a product of two functions, each a solution of the wave equation for $\mathrm{H}_{2}^{+}$.

$$
\begin{gathered}
\psi\left(R ; \boldsymbol{r}_{1}, \boldsymbol{r}_{2}\right)=\chi_{1}\left(R ; \boldsymbol{r}_{1}\right) \chi_{2}\left(R ; \boldsymbol{r}_{2}\right), \\
\left\{-\frac{h^{2}}{8 \pi^{2} m} \nabla_{1}^{2}-\frac{e^{2}}{r_{1}}-\frac{e^{2}}{s_{1}}\right\} \chi\left(R ; \boldsymbol{r}_{1}\right)=\epsilon(R) \chi\left(R ; \boldsymbol{r}_{1}\right) .
\end{gathered}
$$

Now this latter equation is separable in elliptic coordinates $\lambda_{1}=\left(r_{1}+s_{1}\right) / R, \mu_{1}=\left(r_{1}-s_{1}\right) / R, \phi_{1}=$ azimuth about the internuclear axis, and the form of the solutions can be determined in detail. In the cases to be mentioned later the interesting solutions are independent of $\phi$ and can be well approximated by a sum of a few terms of the form $e^{-\delta \lambda} \lambda^{m} \mu^{j}$. This suggests that one might well form the terms of a Ritz function for hydrogen as products of such factors, one for each electron, multiplied by various powers of the interelectronic distance to take account of the tendency of the wave function to decrease as the electrons approach each other: $e^{-\delta\left(\lambda_{1}+\lambda\right)} \lambda_{1}^{m} \lambda_{2}^{n} \mu_{1}^{j} \mu_{2}^{k} r_{12}^{p}$. The symmetry or antisymmetry required of the function according as one treats singlet or triplet states of the system can be assured with only slight complication of the form of the terms: $e^{-\delta\left(\lambda_{1}+\lambda_{2}\right)}\left(\lambda_{1}^{m} \lambda_{2}^{n} \mu_{1}^{j} \mu_{2}^{k} \pm \lambda_{1}^{n} \lambda_{2}^{m} \mu_{1}^{k} \mu_{2}^{j}\right) r_{12}^{p}$. Coolidge and $\mathrm{I}^{13}$ have used just such terms in treating the ground state of $\mathrm{H}_{2}$. For application to excited states of the molecule only one slight modification is necessary. Since the electrons are no longer

${ }^{13}$ H. M. James and A. S. Coolidge, Journal of Chemical Physics, vol. 1 (1933), p. 825 ; ibid., vol. 3 (1935), p. 129. 
equivalent, one must introduce two different exponential parameters for the two electrons, corresponding to the different effective nuclear charges.

In working with these functions we have made extensive studies of the way in which the computed energy converges on the correct energy as more and more terms are added to the Ritz function. The convergence behavior of course varies with the chosen form of the terms, but when a physically appropriate choice has been made the convergence follows a remarkably similar pattern, whether one is working with some state of the hydrogen molecule, or the helium or lithium atoms, or the lithium molecule $\mathrm{Li}_{2}$, or any other system with which we have had any experience.

In a favorable case, of which the ground state of $\mathrm{H}_{2}$ is typical, the convergence is very simple and regular. A presentable approximate function and energy value can be obtained with relatively few terms - say five or six. Thereafter one finds that the small improvements obtained on adding further terms to the function are nearly additive. Thus a term which produces an improvement of .01 volt in the computed energy when it is added to a five-term function will give nearly the same improvement when added, separately or together with other terms, to a ten-term function. Deviations from strict additivity of improvements are almost invariably in the direction of a reduction in the importance of a term when it is added to a better function. Thus it is possible to put an upper limit on the error arising from neglect of any given term by trying it out together with a small number of others. This greatly reduces the labor of eliminating useless terms from consideration and getting the simplest possible Ritz function of a given accuracy.

Another important feature of the convergence is the rapid and regular falling off in importance of the terms in a sequence in which the value of any single exponent is increased. Thus in one case the term with exponents $(0,0,1,0,0)$ was one of the most important terms, $(0,1,1,0,0)$ gave an improvement of .008 electron volts, and $(0,2,1,0,0)$ an improvement of .0015 electron volts. This rapid convergence makes it relatively easy to estimate closely the improvement in energy to be obtained on adding to the Ritz function all the terms of a given sequence. The near independence of the improvements due to the different sequences then makes possible a useful estimate of the convergence limit as all terms of the complete set are added to the Ritz function. For example, we have obtained the following results in treating the ground state of $\mathrm{H}_{2}$. A five-term function gave the energy of the molecule as -2.3329 atomic units, against the observed value 
of -2.3493 - a result already far more accurate than has been obtained by any method other than the Ritz method. A thirteen-term function gave the energy as -2.3470 , correct to $1 / 10$ per cent; the root-mean-square error in that function itself is of the order of 1 per cent. Finally, a careful convergence estimate led to the energy value $-2.3492 \pm .0008$. When this value was published it was considerably more reliable than any available experimental result, but a later very accurate determination by Beutler and Jünger ${ }^{14}$ gave the experimental value quoted above, in complete agreement with the apparent convergence limit of the Ritz method.

In treating some of the excited states of $\mathrm{H}_{2}$ we have encountered a less simple convergence behavior. ${ }^{15}$ Sometimes it happens that two terms will cooperate; that is, they will together improve the computed energy by more than the sum of the improvements they would produce separately. We have not encountered any case in which three or more terms have formed a cooperating group. When such cooperation occurs it is usually easy to understand in physical terms. In practice in treating a given problem one quickly finds out whether such cooperation is likely to occur and can predict fairly accurately what pairs of terms will show it. Somewhat more care is required in discarding terms and making convergence estimates, but the difficulty is not serious.

Somewhat more annoying is the general tendency toward slower convergence in the case of the excited states. With the ground state five terms suffice to give a very useful function, while with an excited state ten terms may be necessary to obtain similar accuracy. This increasing slowness of convergence is most marked when one deals with the higher states of a given symmetry. Thus our best function for the ground state, a ${ }^{1} \Sigma_{o}$ state, contains thirteen terms. To get a somewhat less accurate result for the first excited state of this character sixteen terms were needed, and we have never succeeded in getting a usable approximation for the second excited ${ }^{1} \Sigma_{g}$ state, which, incidentally, would be of particular physical interest.

Despite these difficulties the Ritz method has yielded very useful results for several excited states of $\mathrm{H}_{2} .{ }^{15}$ The maximum accuracy for practical work was obtained with functions containing about sixteen terms, after many other terms were tried and discarded as negligible. These functions are accurate to the order of 2 per cent, the computed energies to about 0.1 per cent.

${ }^{14}$ H. Beutler and H.-O. Jünger, Zeitschrift für Physik, vol. 101 (1936), p. 304.

${ }^{15}$ H. M. James, A. S. Coolidge and R. D. Present, Journal of Chemical Physics, vol. 4 (1936), p. 187; A. S. Coolidge and H. M. James, ibid., vol. 6 (1938), p. 730. 
The physical significance of such applications of the Ritz method may be illustrated by the results of a computation in which use was made of these wave functions. ${ }^{16}$ When $\mathrm{H}_{2}$ is bombarded with electrons having more than 12 electron volts of energy it emits a strong continuous spectrum in the ultraviolet. This arises when molecules excited to the stable $1 s \sigma 2 s \sigma{ }^{3} \Sigma_{o}$ state pass over into the lower and unstable $1 s \sigma 2 p \sigma^{3} \Sigma_{u}$ state. If the energy of the bombarding electrons is carefully kept to a minimum or if enough helium is present in the discharge this spectrum takes on a particularly simple and reproducible character, for the vibrations of the molecule in the initial electronic state are suppressed. Using Ritz wave functions for both electronic states of the molecule we have made a complete theoretical prediction of the intensities in this spectrum. The results appear in Figure 1, together with the observational results of Smith, the best

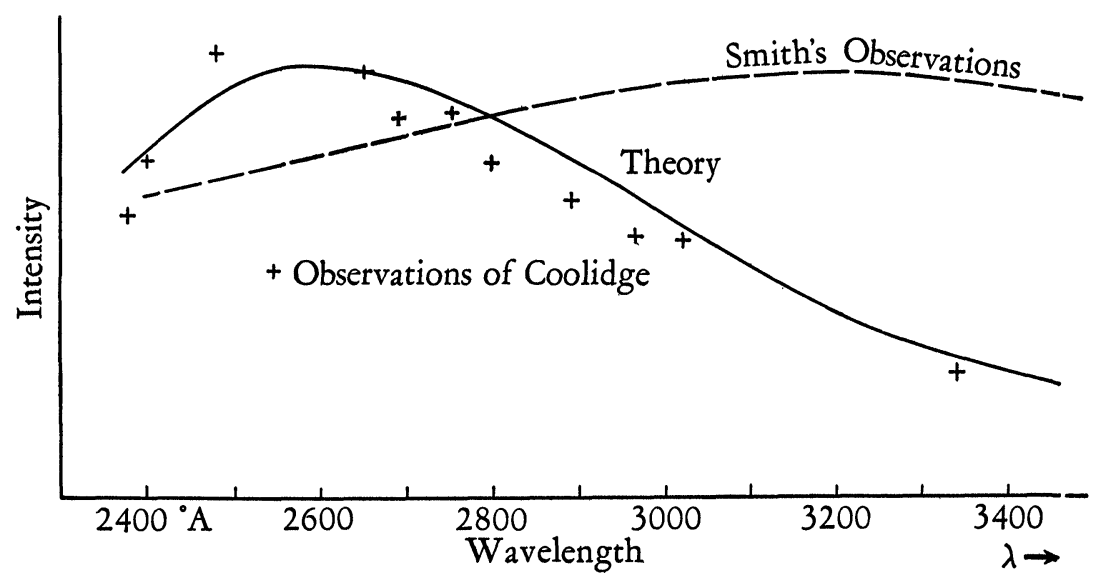

FIG. 1

available at the time. The agreement was not to be regarded as satisfactory. Since we had complete confidence in the theory we were forced to the conclusion that the experiments were in error, and that the intensity standards used by Smith were not satisfactory for work in this very difficult region of the spectrum. This has now been proved to be correct by the preliminary results of some very careful experimental work by Dr. Coolidge, also shown in Figure 1. On employing improved intensity standards, he finds that the gross disagreement of theory with experiment disappears. It is true that there remain discrepancies which are larger than one would like. This seems to be due

${ }^{16}$ H. M. James and A. S. Coolidge, Physical Review, vol. 55 (1939), p. 184. 
to difficulties with the intensity standard, which is still less reliable than the theoretical computations. It would not be at all unreasonable to work backward, from the theory and the observations of Coolidge, to an improved calibration of the standard source.

Now a somewhat less satisfactory aspect of this subject must be mentioned. While the helium atom and the hydrogen molecule are of rather special interest to physicists they are also almost the simplest systems of their respective types, much too simple to be entirely typical. Unfortunately one soon finds on attempting to extend the Ritz method to the treatment of other atomic systems that the difficulties are numerous and serious. To indicate their character I need only to pass to the case of lithium.

The lithium atom consists of a nucleus and three electrons. In the simplest possible case the wave functions depend on six essential and non-separable variables-say the six distances between pairs of particles. The first discouraging effect of this greater number of variables is the corresponding increase in the number of potentially important Ritz terms with all exponents small. In passing from the hydrogen molecule with five essential variables to the lithium atom with six there is actually an increase from eight to thirty-two of the Ritz terms in which no exponent exceeds 1 . The number of sequences of terms to be investigated in making convergence estimates is correspondingly increased.

Each term is also more difficult to work with. The increased number of electrons makes the symmetry conditions more complicated, ${ }^{17}$ so that each Ritz term is, at the simplest, the sum of six parts, instead of the two parts needed with helium or hydrogen. There are thus more integrals to be evaluated in setting up the secular equation for the problem, and they are more difficult to evaluate because of the greater number and the awkward character of the variables of integration.

In the case of lithium it is possible to overcome these difficulties. ${ }^{18}$ The observed total energy of the lithium atom is -14.9578 atomic units. Using a 17-term function we have computed the energy -14.9566 , in error by one part in 10,000. Of more physical interest, perhaps, is the energy needed to ionize the atom. Here the experimental result is 5.364 e.v., while our convergence estimate leads to the theoretical value $5.363 \pm .007$ e.v.

It does not seem useful, however, to apply the Ritz method in accurate treatments of atoms more complicated than lithium. The diff-

${ }^{17}$ H. M. James and A. S. Coolidge, Physical Review, vol. 55 (1939), p. 873.

${ }^{18}$ H. M. James and A. S. Coolidge, Physical Review, vol. 49 (1936), p. 688. 
culties increase enormously and dampen effectively any interest one may have in this method of studying the systems. In the case of molecules the difficulties are even greater; to give an accurate treatment of the molecule $\mathrm{Li}_{2}$ is much more than twice as difficult as to give a corresponding treatment of the lithium atom. It is true that the Ritz method has been fruitfully applied to the treatment of $\mathrm{LiH},{ }^{19}$ and of $\mathrm{Li}_{2},{ }^{20}$ and especially of the ionized molecules ${ }^{19} \mathrm{LiH}^{+}$and $\mathrm{Li}_{2}+,{ }^{21}$ but here again the limits to the extension of the method seem to have been reached.

In conclusion I should emphasize that this particular field of atomic physics has been discussed, not as a summary of all that has been accomplished in physics using the Rayleigh-Ritz method, but rather as an illustration of how the method is likely to work out in practice where it is applied. Applications have been made in widely varied fields - the theory of nuclear structure, acoustics, the electromagnetic theory of cavity resonators - to mention three. The field I have discussed is typical in many respects. One can expect in general that the Ritz method can yield extremely accurate results with some problems not tractable to other methods of computation, and that its application will become impracticable just in cases where very many independent variables must be considered, or boundary or other conditions are especially complicated. What is abnormal about this field is the relative completeness with which the Ritz method has been exploited in it. This is due just to the scarcity of atomic problems in which one can deal with a reasonably small number of independent variables. In the majority of fields one can expect to find an unending sequence of problems to which the method can be usefully applied.

\section{Purdue University}

\footnotetext{
${ }^{19}$ J. K. Knipp, Journal of Chemical Physics, vol. 4 (1936), p. 300.

${ }^{20}$ H. M. James, Journal of Chemical Physics, vol. 2 (1934), p. 794.

${ }^{21}$ H. M. James, Journal of Chemical Physics, vol. 3 (1935), p. 9.
} 